\title{
Laparoscopic Distal Pancreatectomy Preserving the Spleen and Splenic Vessels: An Operation Without Increased Morbidity
}

\author{
Kazuhiro Suzumura*, Kenjiro Iida, Hideaki Iwama, Yusuke Kawabata \\ Department of Surgery, Hyogo College of Medicine, Hyogo, Japan \\ Email address: \\ ssuzumu@hyo-med.ac.jp (K. Suzumura) \\ *Corresponding author \\ To cite this article: \\ Kazuhiro Suzumura, Kenjiro Iida, Hideaki Iwama, Yusuke Kawabata. Laparoscopic Distal Pancreatectomy Preserving the Spleen and Splenic \\ Vessels: An Operation Without Increased Morbidity. Journal of Surgery. Vol. 7, No. 4, 2019, pp. 110-113. doi: 10.11648/j.js.20190704.15
}

Received: June 7, 2019; Accepted: July 15, 2019; Published: August 5, 2019

\begin{abstract}
Background: The indications for laparoscopic spleen-preserving distal pancreatectomy (LSPDP) and the associated morbidity in comparison to laparoscopic distal pancreatectomy with splenectomy (LDPS) are ill-defined. Objective: This study aimed to evaluate the safety, feasibility and outcomes of LSPDP. Methods: Between January 2010 and May 2014, 13 patients underwent LDPS or LSPDP in our institution, and their clinical data and the outcomes of the two procedures were retrospectively reviewed and statistically analyzed. Results: There were no significant differences in age, gender, American Society of Anesthesiologists (ASA) score, body mass index (BMI) or blood loss between the LDPS and LSPDP groups. The mean tumor size ( 8.63 vs. $2.51 \mathrm{~cm}, \mathrm{P}<0.005$ ) and mean operative time ( 353 vs. 235 minutes, $\mathrm{P}<0.029$ ) were greater in the LDPS group than in the LSPDP group. The rates of complications in the two groups did not differ to a statistically significant extent. All of the patients were achieved R0 resection and no mortality. Conclusion: LSPDP with conservation of the splenic artery and vein was a safe and feasible option for benign or low-grade malignant tumors in the distal pancreas, because of no mortality, no splenic infarction and R0 resection. Splenic conservation does not significantly increase the morbidity associated with the procedure.
\end{abstract}

Keywords: Pancreas, Laparoscopy, Distal Pancreatectomy, Spleen-preserving, Splenic Vessels

\section{Introduction}

Laparoscopic surgery has been developed to the extent that it is now the standard technique for treating many conditions [1-5]. Although the number of laparoscopic distal pancreatectomies performed is still low, laparoscopic distal pancreatectomy has become the minimally invasive procedure of choice for treating pancreatic disease. Laparoscopic distal pancreatectomy is accepted for benign and low-grade malignant tumors located in the distal pancreas [6-10]. However, irrespective of whether distal pancreatectomy is performed by open or laparoscopic procedures, the indications for spleen preservation are ill-defined. Whether splenectomy can cause a series of adverse consequences, such as postoperative abscess and sepsis has been widely debated in the literature and remains controversial [11-14]. Furthermore, although there have been many comparisons of open procedures, few reports have extensively compared laparoscopic spleen-preserving distal pancreatectomy (LSPDP) to laparoscopic distal pancreatectomy with splenectomy (LDPS). The purpose of this study was to compare the safety and outcomes of LSPDP to those of LDPS.

\section{Patients and Methods}

\subsection{Patients}

Between January 2010 and May 2014, 13 patients underwent LDPS or LSPDP at Hyogo College of Medicine. Only patients with a preoperative diagnosis of benign or low-grade malignant tumor by CT or MRI were considered for laparoscopic surgery. The medical records of these patients were retrospectively reviewed to collect the following data: patient characteristics, preoperative investigations, operative procedures, splenic preservation, tumor size, blood loss, postoperative complications, pathologic diagnosis, and outcome. 


\subsection{Surgical Procedures}

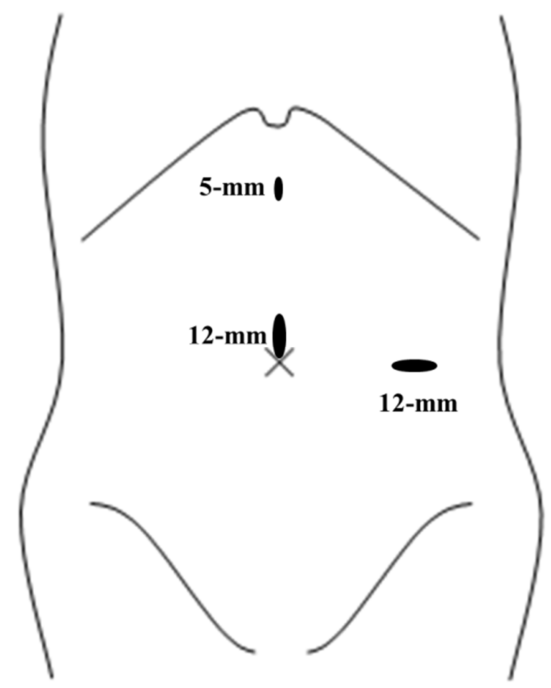

Figure 1. Port placement for laparoscopic distal pancreatectomy.

Under general anesthesia, the patients were placed in the right semilateral position. Three trocars were then placed: (1) the middle umbilical site for laparoscopy (12-mm), (2) the epigastric margin as a working trocar (5-mm), and (3) the left lateral abdomen on the anterior axillary line as a working trocar (12-mm) (Figure 1).

LDPS was indicated for severe adhesion between the pancreatic tail and spleen. During LSPDP, the splenocolic ligament was divided using laparoscopic coagulation shears, then the splenic flexure of the colon was detached toward the caudal side. The omental bursa was opened so that the anterior surfaces of the pancreatic body and tail could be adequately visualized. The dissection was performed within the avascular plane along the posterior surface of the pancreas from the inferior border. Using a vessel-sealing device, many branches of the splenic vessels were divided from the pancreatic tail toward the body, and the distal pancreas was mobilized. The pancreatic parenchyma was transected with an endoscopic linear stapler (60-mm in length and 4.8-mm in staple height), which allowed an adequate margin from the lesion (Figure 2a, b). After enlarging the middle umbilical site port, the specimen was removed from the abdominal cavity using an endoscopic bag retrieval system. A closed drain was placed at the stump of the pancreas.

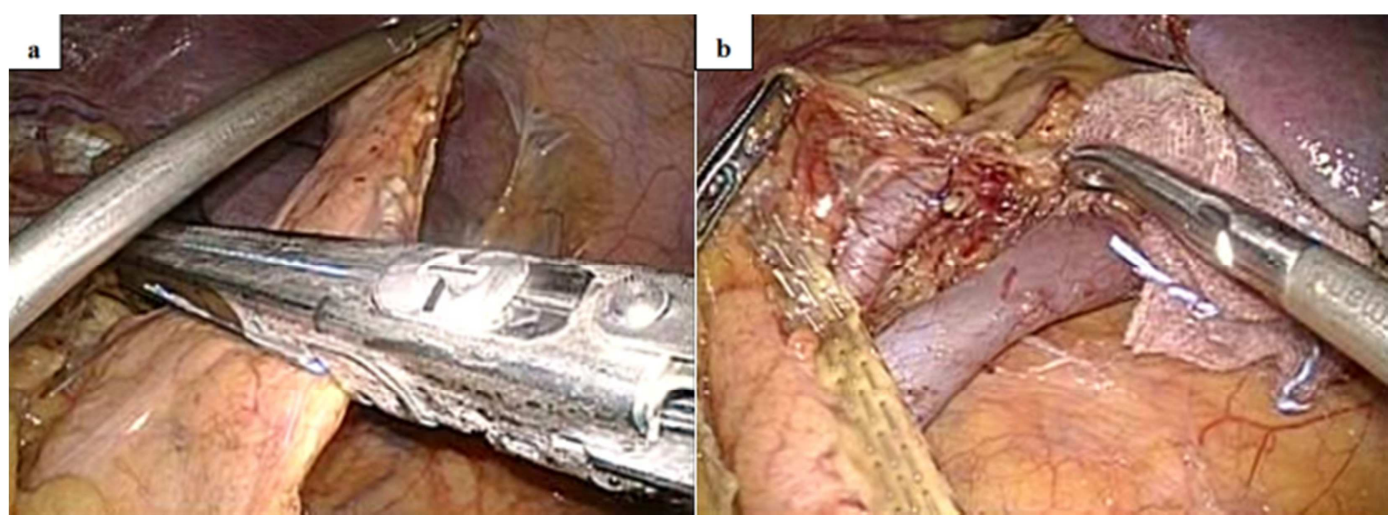

Figure 2. $a$. The distal pancreas is transected with an endoscopic linear stapler; $b$. After laparoscopic distal pancreatectomy preserving the spleen and splenic vessels.

\subsection{Pancreatic Fistula}

Pancreatic fistula was classified as either biochemical (transient fistula) or clinical. Biochemical fistula was defined by a drain amylase level of more than three times the normal serum activity after postoperative day (POD) 3. Clinical fistula was defined according to the 2005 International Study Group of Pancreatic Fistulas [15], and was classified into grades $\mathrm{B}$ and $\mathrm{C}$.

\subsection{Statistical Analysis}

Data were presented as the mean $\pm \mathrm{SD}$. The chi-squared test or Fisher's exact test were used for the analysis of categorical variables, as appropriate. $P$ values of $<0.05$ were considered to indicate statistical significance.

\section{Results}

A total of 13 distal pancreatectomies were completed laparoscopically. The 13 patients were included 4 males and 9 females with a mean age of $50 \pm 18$ years (range, 14-76 years). LDPS and LSPDP were performed in 3 and 10 patients respectively. All patients were found to have a mass in the body or tail of the pancreas by CT or MRI and were preoperatively diagnosed as having benign or low-grade malignant tumors.

The preoperative and intraoperative data are shown in Table 1.

Table 1. The preoperative and intraoperative data of the LDPS and LSPDP groups.

\begin{tabular}{llll}
\hline Parameter & LDPS & LSPDP & $\boldsymbol{P}$ value \\
\hline Male/Female & $0 / 3$ & $4 / 6$ & 0.188 \\
Age & $33 \pm 16$ & $55 \pm 16$ & 0.057 \\
ASA score & $1.3 \pm 0.6$ & $1.9 \pm 0.9$ & 0.322 \\
Body mass index & $20.3 \pm 3.5$ & $21.5 \pm 3.2$ & 0.606 \\
Tumor size (cm) & $8.63 \pm 4.3$ & $2.51 \pm 2.08$ & 0.005 \\
Operative time (min) & $353.0 \pm 107$ & $234.7 \pm 61.1$ & 0.029 \\
Blood loss (mL) & $53.3 \pm 41.6$ & $24.5 \pm 22.8$ & 0.136 \\
\hline
\end{tabular}

LDPS: laparoscopic distal pancreatectomy with splenectomy, LSPDP: laparoscopic spleen-preserving distal pancreatectomy, ASA: American society of anesthesiologist. 
The mean American Society of Anesthesiologists (ASA) score was $1.8 \pm 0.8$ (range $1-3$ ), the mean body mass index (BMI) was 21.2 \pm 3.1 (range 16.4-27.7), the mean tumor size was $3.9 \pm 3.7 \mathrm{~cm}$ (range $0.9-13$ ), the mean operative time was $262 \pm 86$ minutes (range 138-474), and the mean blood loss was $31.2 \pm 29 \mathrm{~mL}$ (range 10-100). In the LDPS and LSPDP groups, there were no significant differences in gender, age, ASA score, BMI or blood loss; however, in the LDPS group, the tumor size was larger and the operative time was longer in comparison to the LSPDP group.

The postoperative data are shown in Table 2.

Table 2. Postoperative complications in the LDPS and LSPDP groups.

\begin{tabular}{llll}
\hline Parameter & LDPS & LSPDP & $\boldsymbol{P}$ value \\
\hline Pancreatic fistula & 1 & 8 & 0.125 \\
Clinical pancreatic fistula (grade B, C) & 1 & 2 & 0.631 \\
Postoperative bleeding & 0 & 0 & - \\
Abdominal infection & 1 & 2 & 0.34 \\
Splenic infarction & 0 & 0 & - \\
Portal system thrombosis & 1 & 0 & 0.057 \\
Perioperative mortality & 0 & 0 & - \\
\hline
\end{tabular}

Pancreatic fistula was the most frequent postoperative complication. Based on the ISGPF definition, clinical pancreatic fistula developed in 1 patient in the LDPS group and 2 patients in the LSPDP group. Other complications included abdominal infection and portal system thrombosis in the LDPS group and abdominal infection in the LSPDP group. The incidence of these complications in the two groups did not differ to a statistically significant extent. Moreover there was no splenic infarction or mortality.

The final pathological diagnoses are shown in Table 3.

Table 3. Tumor characteristics.

\begin{tabular}{llll}
\hline Pathology & $\begin{array}{l}\text { LDPS } \\
(\%)\end{array}$ & $\begin{array}{l}\text { LSPDP } \\
(\%)\end{array}$ & $\begin{array}{l}\text { Total } \\
(\%)\end{array}$ \\
\hline Neuroendocrine tumor & $0(0)$ & $6(60)$ & $6(46.1)$ \\
Mucinous cystic neoplasm & $2(66.7)$ & $0(0)$ & $2(15.4)$ \\
Solid pseudopapillary neoplasm & $1(33.3)$ & $1(10)$ & $2(15.4)$ \\
Intrapancreatic accessory spleen & $0(0)$ & $2(20)$ & $2(15.4)$ \\
True cyst & $0(0)$ & $1(10)$ & $1(7.7)$ \\
\hline
\end{tabular}

All patients were pathologically diagnosed and R0 resection was confirmed. All of the patients remained alive without recurrence over a median follow-up period of 25 months.

\section{Discussion}

Laparoscopic distal pancreatectomy has become the minimally invasive procedure of choice for pancreatic disease. In the past, surgeons preferred to remove the spleen simultaneously because the intimate relationship between the splenic vessels and the pancreas makes separation difficult. In recent years, however, the immunological role of the spleen has been increasingly emphasized and many adverse consequences have been reported after splenectomy. In distal pancreatectomy, splenectomy is reported to be associated with long-term risk of postsplenectomy sepsis related to encapsulated bacteria [16].
Thus, spleen-preservation is desirable.

There are two distinct approaches to spleen-preservation during the dissection of the distal pancreas. The classic approach is to identify, isolate, and preserve the splenic artery and vein [17]. Alternatively, Warshaw described a spleen-preserving approach in which the splenic artery and vein are ligated with the pancreas, and perfusion of the spleen is maintained by the short gastric vessels [14]. Many authors have reported serious complications when using Warshaw's technique, including necrosis of the entire spleen or abscess necessitating re-operation for splenectomy [18-21]. Thus, we perform laparoscopic distal pancreatectomy preserving the spleen and splenic vessels.

Many clinical trials and case series have compared open distal pancreatectomy with or without spleen preservation. Most studies documented a remarkable decrease in postoperative infection and complications in spleen-preservation groups in comparison splenectomy groups [11-13], whereas others had contradictory findings [14, 16]. To our knowledge, few reports have concentrated on such comparisons of laparoscopic procedures. In our study, there were no significant differences in gender, age, ASA score, BMI or blood loss between the LDPS and LSPDP groups. In the LSPDP group, the tumor size was smaller and operative time was shorter in comparison to the LDPS group.

There were no significant differences between the two groups with regard to the incidence of postoperative complications. Pancreatic fistula is the most serious complication. Management of the pancreatic stump and prevention of pancreatic fistula have long been concerns for pancreatic and endoscopic surgeons. A recent meta-analysis of sutured versus stapled closure confirmed that stapled resection tended to be associated with fewer leaks; however, the results did not reach statistical significance [16].

We have preferentially used a 4.8- $\mathrm{mm}$ endoscopic linear staple in laparoscopic distal pancreatectomy. The total clinical pancreatic fistula rate was $23 \%$, and pancreatic fistulas were cured by drainage and antibiotics, one patient in the LDPS group and 2 in the LSPDP group developed clinical pancreatic fistulas.

\section{Conclusion}

Our results showed that LSPDP with conservation of the splenic artery and vein was a safe and feasible option for benign or low-grade malignant tumors in the distal pancreas, because of no mortality, no splenic infarction and $\mathrm{R} 0$ resection. Splenic conservation does not significantly increase the morbidity associated with the procedure.

\section{References}

[1] Guillou PJ, Quirke P, Thorpe H, Walker J, Jayne DG, Smith $\mathrm{AM}$, et al. Short-term endpoints of conventional versus laparoscopic-assisted surgery in patients with colorectal cancer (MRC CLASICC trial): multicenter randomized controlled trial. Lancet. 2005; 365: 1718-1726. 
[2] Kitano S, Shiraishi N, Uyama I, Sugihara K, Tanigawa N. A multicenter study on oncologic outcome of laparoscopic gastrectomy for early cancer in Japan. Ann Surg. 2007; 245: 68-72.

[3] Habermaiz B, Sauerland S, Deker G, Delaitre B, Gigot JF, Leandros E, et al. Laparoscopic splenectomy: the clinical practice guidelines of the European Association for Endoscopic Surgery (EAES). Surg Endosc. 2008; 22: 821-848.

[4] Miyajima N, Fukunaga M, Hasegawa H, Tanaka J, Okuda J, Watanabe M. Results of a multicenter study of 1,057 cases of rectal cancer treated by laparoscopic surgery. Surg Endosc. 2009; 23: 113-118.

[5] Buell JF, Cherqui D, Geller DA, O'Rourke N, Iannitti D, Dagher I, et al. The international position on laparoscopic liver surgery: the Louisville Statement, 2008. Ann Surg. 2009; 250: 825-830.

[6] Karaliotas C, Sgourakis G. Laparoscopic versus open enucleation for solitary insulinoma in the body and tail of the pancreas. J Gastrointest Surg. 2009; 13: 1869.

[7] Melotti G, Butturini G, Piccoli M, Casetti L, Bassi C, Mullineris B, et al. Laparoscopic distal pancreatectomy: results on a consecutive series of 58 patients. Ann Surg. 2007; 246: 77-82.

[8] Borja-Cacho D, Al-Refaie WB, Vickers SM, Tuttle TM, Jensen EH. Laparoscopic distal pancreatectomy. J Am Coll Surg. 2009; 209: 758-765.

[9] Teh SH, Tseng D, Sheppard BC. Laparoscopic and open distal pancreatic resection for benign pancreatic disease. J Gastrointest Surg. 2007; 11: 1120-1125.

[10] Mabrut JY, Fernandez-Cruz L, Azagra JS, Bassi C, Delvaux G, Weerts J, et al. Laparoscopic pancreatic resection: results of a multicenter European study of 127 patients. Surgery. 2005; 137: 597-605.

[11] Shoup M, Brennan MF, McWhite K, Leung DH, Klimstra D, Conlon KC. The value of splenic preservation with distal pancreatectomy. Arch Surg. 2002; 137; 164-168.
[12] Dulucq JL, Wintringer P, Stabilini C, Feryn T, Perissat J, Mahajna A. Are major laparoscopic pancreatic resections worthwhile? A prospective study of 32 patients in a single institution. Surg Endosc. 2005; 19: 1028-1034.

[13] Carrère N, Abid S, Julio CH, Bloom E, Pradère $\mathrm{B}$. Spleen-preserving distal pancreatectomy with excision of splenic artery and vein: a case-matched comparison with conventional distal pancreatectomy with splenectomy. World J Surg. 2007; 31: 375-382.

[14] Rodríguez JR, Madanat MG, Healy BC, Thayer SP, Warshaw AL, Fernández-del Castillo C. Distal pancreatectomy with spleen preservation revisited. Surgery. 2007; 141: 619-625.

[15] Bassi C, Dervenis C, Butturini G, Fingerhut A, Yeo C, Izbicki J, et al. Postoperative pancreatic fistula: an international study group (ISGPF) definition. Surgery. 2005; 138: 8-13.

[16] Holdsworth RJ, Irving AD, Cuschieri A. Postsplenectomy sepsis and its mortality rate: actual versus perceived risks. Br J Surg. 1991; 78: 1031-1038.

[17] Bruzoni M, Sasson AR. Open and laparoscopic spleen-preserving, splenic vessel-preserving distal pancreatectomy: indications and outcomes. J Gastrointest Surg. 2008; 12: 1202-1206.

[18] Vezakis A, Davides D, Larvin M, McMahon MJ. Laparoscopic surgery combined with preservation of the spleen for distal pancreatic tumors. Surg Endosc. 1999; 13: 26-29.

[19] Shimizu S, Tanaka M, Konomi H, Tamura T, Mizumoto K, Yamaguchi K. Spleen-preserving laparoscopic distal pancreatectomy after division of the splenic vessels. J Laparoendosc Adv Surg Tech A. 2004; 14:173-177.

[20] Fernández-Cruz L, Martínez I, Gilabert R, Cesar-Borges G, Astudillo E, Navarro S. Laparoscopic distal pancreatectomy combined with preservation of the spleen for cystic neoplasms of the pancreas. J Gastrointest Surg. 2004; 8: 493-501.

[21] Pryor A, Means JR, Pappas TN. Laparoscopic distal pancreatectomy with splenic preservation. Surg Endosc. 2007; 21: 2326-2330. 